\title{
FACTORIZATIONS OF FREE ACTIONS OF FINITE GROUPS ON COMPACT $Q$-MANIFOLDS
}

\author{
VO-THANH-LIEM
}

\begin{abstract}
We show that every free action of a finite group $G$ on a compact $Q$-manifold $K^{k} \times Q$ is conjugate to the product of a $G$-free action on a regular neighborhood of $K$ in $\mathbf{R}^{2 k+1}\left(\mathbf{R}^{7}\right.$, if $\left.k=2\right)$ and the identity on $Q$. Also, sharper results for special finite complexes $K$ are obtained.
\end{abstract}

1. Free action of finite cyclic groups on $S^{n} \times Q$. Two free actions of a group $G$ on a topological space $M$ are conjugate if there is a homeomorphism $f$ of $M$ onto itself such that $f \circ \lambda_{g} \circ f^{-1}=\lambda_{g}^{\prime}$, where $\lambda_{g}$ and $\lambda_{g}^{\prime}$ are correspondent homeomorphisms of $M$ defined by any element $g$ of $G$.

One wonders which free action of a finite group $G$ on $S^{n} \times Q$ is conjugate to the product of a $G$-free action on the sphere $S^{n}$ and the identity on the Hilbert cube $Q$; or equivalently, are their orbit spaces homeomorphic? By use of Chapman $\left[\mathrm{Ch}_{1}\right]$ and West [We $\left[\mathrm{W}_{1}\right]$ these are equivalent to showing that the orbit space of the given free action, compact $Q$-manifold, is simple homotopy equivalent to a compact topological $n$-manifold without boundary.

In this section, we will prove the following theorems.

THEOREM 1. Every $Z_{2}$-free action on $S^{n} \times Q$ is conjugate to the product of the standard $\mathrm{Z}_{2}$-free action on $S^{n}$ and the identity on $Q$.

TheOREM 2. For $p>2$, every $\mathrm{Z}_{p}$-free action on $S^{2 n-1} \times Q$ is conjugate to the product of a $\mathbf{Z}_{p}$-free action on $S^{2 n-1}\left(S^{3} \times I^{2}\right.$, if $\left.n=2\right)$ and the identity on $Q$.

It is interesting to mention that a similar statement is not true for arbitrary finite groups.

Let $N$ be a Poincaré complex whose fundamental group is the symmetric group $S_{3}$ and whose universal covering space $\tilde{N}$ has the homotopy type of the 3-sphere $S^{3}$. (Such a complex exists by the second part of Theorem 4.3 in $\left[\mathbf{W}_{1}\right]$.) Moreover, since the obstruction to $N$ being of finite type lies in $\tilde{K}^{0}\left(S_{3}\right)=0\left[W_{2}\right.$, p. 67], we may assume that $N$ is a finite complex.

Now, Theorem 5.2 of [We] or Lemma 4.2 of [Ch $]$ shows that $\tilde{N} \times Q \approx S^{3}$ $\times Q$. Therefore, an exotic free action of $S_{3}$ on $S^{3} \times Q$ is obtained naturally,

Presented to the Society, December 5, 1977; received by the editors February 7, 1977 and, in revised form, June 13, 1978.

AMS (MOS) subject classifications (1970). Primary 57E25; Secondary 57A20, 58B05.

Key words and phrases. Hilbert cube manifold, lens space, Whitehead torsion, Poincare complex, $h$-cobordism, regular neighborhood, covering space. 
since $S_{3}$ cannot act freely on $S^{3}$ ([M]). For large $n$, exotic free actions of $S_{3}$ on $S^{n} \times Q$ can also be similarly obtained.

For the notion of finite Poincaré complex, we refer to Chapter 2 of [ $\left.\mathbf{W}_{3}\right]$.

Proof of Theorem 2. For $n=1$, it is trivial. So, we assume $n>2$.

Step 1. The orbit space $\left(S^{2 n-1} \times Q\right) / Z_{p}$ is homotopy equivalent to a lens space $L_{n}(p ; q, 1, \ldots, 1)$ for some $q$.

Let $N$ be a finite complex such that $N \times Q \approx\left(S^{2 n-1} \times Q\right) / Z_{p}$. Then $N$ is a $\left(Z_{p}, 2 n-1\right)$-polarisation (Definition 2.1 of [T-W]), and by Remark 2.6 of [T-W], it is a finite Poincaré complex.

Now, by Theorem 4E.3 of $\left[\mathrm{W}_{3}\right]$, we may assume that $N=K \cup_{f} e^{2 n-1}$, where $K$ is the $(2 n-2)$-skeleton of the lens space $L_{n}(p ; 1, \ldots, 1)$. Hence, the fibration $\mathbf{Z}_{p} \rightarrow \tilde{N} \rightarrow N$ is classified by a map $\varphi: N \rightarrow L_{n}(p ; 1, \ldots, 1)$. (For notation of lens space refer to $\left[\mathrm{Co}_{1}\right]$.)

It is clear that the induced map $\varphi_{\#}$ on fundamental groups is an isomorphism. We claim that the degree $a$ of $\varphi$ is relatively prime to $p$. Indeed, let $\alpha$ be a generator of $\pi_{1}(N)=\mathbf{Z}_{p}$. By use of the cell-structure of $L_{n}(p ; 1, \ldots, 1)$ (refer to $\left.\left[\mathrm{Co}_{1}\right]\right)$, we can define skeleton-wise a map $\psi$ from $L_{n}(p ; 1, \ldots, 1)$ to $N$ such that $\psi_{\#}\left(\varphi_{\#}(\alpha)\right)=\alpha$; hence, $(\varphi \circ \psi)_{\#}$ is the identity on $\pi_{1}\left(L_{n}(p ; 1, \ldots, 1)\right)$. Now, (29.4) of [Co $]$ shows that the degree of $\varphi \circ \psi \equiv 1$ $(\bmod p)$. So, the claim is proved.

Again, by (29.4) of $\left[\mathrm{Co}_{1}\right]$, we have a map $\theta: L_{n}(p ; 1, \ldots, 1) \rightarrow$ $L_{n}(p ; q, 1, \ldots, 1)$ of degree $q$ inducing an isomorphism on fundamental groups, where $q$ is an integer such that $a q \equiv 1(\bmod p)$.

Now, using the cell-structure of $N=K \cup_{f} e^{2 n-1}$ to modify the map $\theta \circ \varphi$ over a closed ball in Int $e^{2 n-1}$ (see [Co, p. 95]), we may obtain a map $\mu$ : $N \rightarrow L_{n}(p ; q, 1, \ldots, 1)$ such that

(a) $\mu_{\#}$ is an isomorphism on fundamental groups,

(b) degree $\mu=1$.

Then, it is routine to show that $\mu$ is a homotopy equivalence. The proof of Step 1 is complete.

Step 2. Case 1. $(2 n-1>3)$.

Let $\left(W ; L_{n}(p ; q, 1, \ldots, 1), M\right)$ be a PL $h$-cobordism such that the torsion $\tau\left(W, L_{n}(p ; q, 1, \ldots, 1)\right)=\tau\left(\mu^{-1}\right)$, the torsion of the homotopy equivalence $\mu^{-1}$ in Step 1 [H, Theorem 12.1]. Then $N$ and $M$ have the same simple homotopy type. The proof of this case is complete.

Case 2. $(2 n-1=3)$. Let $\left(W^{6} ; L_{2}(p ; q, 1) \times I^{2}, M^{5}\right)$ be a suitable $h$ cobordism as in Case 1 such that $\partial W=\left[L_{2}(p ; q, 1) \times I^{2}\right] \cup M^{5} \cup$ $\left[L_{2}(p ; q, 1) \times \partial I^{2} \times I\right]$. In particular $\partial M=L_{2}(p ; q, 1) \times \partial I^{2}$.

Now, it is clear that $\left(\tilde{W}^{6} ; \tilde{L}_{2}(p ; q, 1) \times I^{2}, \tilde{M}^{5}\right)$ is also an $h$-cobordism (it is trivial on $\left.\tilde{L}_{2}(p ; q, 1) \times S^{1} \times I\right)$. Moreover, since $\pi_{1}\left(\tilde{W}^{6}\right)=0$, we have, by the relative $h$-cobordism theorem ([R-S, Theorem 6.18])

$$
\tilde{M}^{5} \approx \tilde{L}_{2}(p ; q, 1) \times I^{2}=S^{3} \times I^{2} \text {. }
$$

Therefore, the proof of Theorem 2 is complete. 
Proof of Theorem 1. Using notation of Step 1 above, $N$ is also a finite Poincaré complex of formal dimension $n$, by Remark 2.6 of [T-W]. Then, $N \approx P_{n}(\mathbf{R})$ by use of $(4.3)$ of $\left[\mathbf{W}_{3}\right]$ or by going along the proof of Step 1 above.

Finally, the proof of the theorem is complete since the Whitehead group of $\mathbf{Z}_{2}$ is trivial [ $\left.\mathrm{Co}_{\mathbf{1}}\right]$.

2. Free actions of a finite group $G$ on compact $Q$-manifolds. For compact ANR's $X$ and $Y$, let $X \cong Y\left(X \approx_{s} Y\right)$ mean that $X$ and $Y$ have the same (simple) homotopy type.

Given a simplicial complex $X$ and a normal subgroup $H$ of $\pi_{1}\left(X, x_{0}\right)$ such that $G \approx \pi_{1}\left(X, x_{0}\right) / H$ is of finite order, denote by $\left(X(G), e_{0}\right)$ the $G$-covering space of $\left(X, x_{0}\right)$; i.e. the transformation group of the covering map $\left(X(G), e_{0}\right) \underset{p}{\rightarrow}\left(X, x_{0}\right)$ is $G$, and $p_{*} \pi_{1}\left(X(G), e_{0}\right)=H$. It is well known that $X(G)$ is unique up to homeomorphism (see [G, Corollary 6.9]).

Let $\tilde{X}$ be the universal covering space of a finite simplicial complex $X, \Lambda$ the integral group ring $\mathrm{Z}_{1} X$ and $C_{*}(X)$ the cellular chain complex of $\tilde{X}$. As in $\left[\mathbf{W}_{\mathbf{3}}\right]$, we let

$$
\begin{aligned}
& H_{*}^{t}(X ; B)=H\left(C_{*}(X) \otimes_{\Lambda} B\right), \\
& H^{*}(X ; B)=H\left(\operatorname{Hom}\left(C_{*}(X) ; B\right)\right),
\end{aligned}
$$

where $B$ is a (right) $\Lambda$-module.

According to the proof of Theorem $\mathrm{F}$ in $\left[\mathrm{W}_{2}\right]$, the condition $D_{n}$ can be restated as follows:

(i) $H_{i}(\tilde{X} ; \mathbf{Z})=0, i>n$,

(ii) $H^{n+1}(X ; B)=0$ for every $\Lambda$-module $B$.

For an integer $k>1$, we define the integer $q(k)$ as follows

(i) $q(k)=3$ if $k=2$,

(ii) $q(k)=k$ if $k \neq 2$.

The main result of this section is the following theorem.

THEOREM 3. Given a finite complex $K^{k}$, any $G$-free action on the compact $Q$-manifold $K \times Q$ is conjugate to the product of a $G$-free action on a regular neighborhood of $K$ in $\mathbf{R}^{2 q(k)+1}$ and the identity on $Q$.

As we mentioned before, there might be exotic free actions of finite groups on $S^{k} \times Q$. However, since the regular neighborhood of $S^{k}$ in $\mathbf{R}^{m}$ is homeomorphic to $S^{k} \times I^{m-k}$, we have the following factorization.

Corollary. Any $G$-free action on $S^{k} \times Q$ is conjugate to the product of a $G$-free action on $S^{k} \times I^{q(k)}$ and the identity on $Q$.

Before giving a proof for Theorem 3, we need some technical lemmas.

LEMMA 1. Let $G$ act freely and simplicially on a finite complex $Y^{q}$ and the orbit space $X^{q}$. If $N$ is a regular neighborhood of $X$ in $\mathbf{R}^{2 q+1}$, then $N(G)$ is a regular neighborhood of $Y$ in $\mathbf{R}^{2 q+1}$. 
Proof. Let $p: N(G) \rightarrow N$ be the orbit map, then it is obvious that $p$ is a PL-immersion. Approximate $p$ by $\bar{p}$ with $\bar{p}$ being in general position. Then, $\bar{p}$ is also a PL-immersion and $\bar{p} \mid Y$ is a PL-embedding. So there is a small regular neighborhood $U(G)$ of $Y$ in $N(G)$ such that $\bar{p} \mid U(G)$ is a PL-embedding, where $U$ is a small regular neighborhood of $X$ in $N$. So $U(G) \approx N(G)$ by the uniqueness of regular neighborhoods.

LEMMA 2. Let $G$ act freely on a compact $A N R X$. If $X$ has the homotopy type of a finite complex $K^{k}$, then the orbit spaces $Y$ has the homotopy type of a finite complex of dimension $q(k)$.

Proof. It is obvious that $Y$ is a compact ANR. Let $f: Y^{\prime} \rightarrow Y$ be a homotopy equivalence, where $Y^{\prime}$ is a finite complex [We $]$. Then consider the orbit map $p: Y^{\prime}(G) \rightarrow Y^{\prime}$ induced by $f$. So, we may assume that $X$ is a finite complex and the action is simplicial.

Now, we intend to use Theorem $F$ of $\left[\mathrm{W}_{2}\right]$. Since $Y$ is a finite complex, it suffices to show that $Y$ satisfies the condition $D_{n}: H_{i}(\tilde{Y})=0$ for $i>q(k)$ and $H^{q(k)+1}(Y ; B)=0$ for every $Z \pi_{1} Y$-module $B$.

By Lemma 1 above, we may assume that $X$ is a regular neighborhood of a copy of $K$ in $\mathbf{R}^{2 q(k)+1}$; so $(Y, \partial Y)$ is a finite Poincaré complex of formal dimension $2 q(k)+1$.

By general position theorem, it follows that the pair $(X, \partial X)$ is $q(k)$-connected. Then, so is the pair $(Y, \partial Y)$ by the five-lemma and the homotopy lifting property of covering maps.

Now by Lemma 1.1 of $\left[\mathrm{W}_{2}\right]$, the pair $(Y, \partial Y)$ is homotopy equivalent to a pair $\left(Y_{1}, \partial Y\right)$ rel $\partial Y$ such that $Y_{1}-\partial Y$ contains no cells of dimension less than $q(k)+1$. So, it follows that $H_{r}^{t}(Y, \partial Y ; B)=0$ and $H^{r}(Y, \partial Y ; B)=0$ for all $r<q(k)$ and $Z \pi_{1} Y$-module $B$.

Therefore, the Poincaré duality theorem in $\left[\mathrm{W}_{3}\right]$ shows that $H^{r}(Y ; B)=0$ and $H_{r}^{t}(Y ; B)=0$ for all $r \geqslant q(k)+1$ [ $\mathbf{W}_{3}$, Theorem 2.1].

The proof of Lemma 2 is complete.

Proof of Theorem 3. Chapman $\left[\mathrm{Ch}_{1}\right]$ shows that $(K \times Q) / G \approx L \times Q$ for some finite complex $L$. We may assume that $\operatorname{dim} L<q(k)$. For $L \cong L^{\prime}$, $\operatorname{dim} L^{\prime}<q(k)$ by Lemma 2 above. If $q(k)=1$, then $L \approx_{s} L^{\prime}$ since the Whitehead groups of free groups are trivial $\left[\mathrm{Co}_{1}\right.$, Theorem 11.6]. If $q(k)>3$, from Lemma 1.1 of $\left[\mathrm{Co}_{2}\right]$ it follows that, given any torsion $\tau \in \mathrm{Wh}\left(\pi_{1} L^{\prime}\right)$, there is a finite complex $L^{\prime \prime} \supset L^{\prime}$ such that $\operatorname{dim}\left(L^{\prime \prime}-L^{\prime}\right)<3$ and the torsion of the pair $\left(L^{\prime \prime}, L^{\prime}\right)$ is $\tau$. So, there exists a finite complex $L^{\prime \prime}$ of dimension $q(k)$ such that $L^{\prime \prime} \simeq_{s} L$.

Let $N$ be a regular neighborhood of $L(G)$ in $\mathbf{R}^{2 q(k)+1}$ given by Lemma 1 . Then, it is easy to show that $K \cong_{s} N$.

Now, let $f: K \rightarrow$ Int $N \subset N$ be a PL-embedding which defines the simple homotopy equivalence. Let $W$ be a regular neighborhood of $f(K)$ in Int $N$, then $(N-$ Int $W ; \partial W, \partial N)$ is an $h$-cobordism of zero torsion. For it is 
routine to show that the inclusion maps $\partial N \rightarrow(N-$ Int $W)$ and $\partial W \rightarrow(N-$ Int $W)$ are homotopy equivalences; and, furthermore, $\partial W \rightarrow(N-$ Int $W)$ is a simple homotopy equivalence by use of the excision theorem 20.3 in [ $\left.\mathrm{Co}_{1}\right]$ and the functorial property of $G \rightarrow \mathrm{Wh}(G)$ (see [ $\left.\left.\mathrm{CO}_{1}, \mathrm{p} .40\right]\right)$.

Therefore, from the $h$-cobordism theorem it follows easily that $N \approx W$. The proof of the theorem is complete.

The author would like to thank R. D. Anderson and M. Colvin for their interesting discussions, and the referee for his valuable suggestions.

\section{REFERENCES}

[Ch, T. A. Chapman, Triangulating compact Hilbert cube manifolds, (to appear).

$\left[\mathrm{Cl}_{2}\right] \ldots$, Topological invariance of Whitehead torsion, Amer. J. Math. 96 (1974), $488-496$.

[Co,] M. M. Cohen, A course in simple-homotopy theory, Springer-Verlag, New York, 1973.

[C $\left.\mathrm{CO}_{2}\right]$ Whitehead torsion, group extensions, and Zeeman's conjecture in high dimensions, (to appear).

[G] M. Greenberg, Lectures on algebraic topology, Benjamin, New York, 1967.

[H] J. F. P. Hudson, Piecewise linear topology, Benjamin, New York, 1969.

[M] J. Milnor, Groups which act on $S^{n}$ without fixed points, Amer. J. Math. 79 (1957), 623-630.

[R-S] C. P. Rourke and B. J. Sanderson, Introduction to piecewise linear topology, SpringerVerlag, New York, 1972.

[W.] C. T. C. Wall, Poincaré complexes. I, Ann. of Math. (2) 86 (1967), 213-245.

$\left[\mathrm{W}_{2}\right]$ _ Finiteness conditions for CW-complexes, Ann. of Math. (2) 81 (1965), 56-69.

$\left[\mathrm{W}_{3}\right]$, Surgery on compact manifolds, Academic Press, New York, 1970.

[T-W] C. B. Thomas and C. T. C. Wall, The topological spherical space form problem. I, Compositio Math. 23 (1971), 101-114.

[We ] J. E. West, Infinite products which are Hilbert cubes, Trans. Amer. Math. Soc. 150 (1970), 1-25.

[We] , Mapping Hilbert cube manifolds to ANR's: A solution of a conjecture of Borsuk, Ann. of Math. (2) 106 (1977), 1-18.

Department of Mathematics, Louisiana State University, Baton Rouge, Louisiana 70803 